\title{
Health Care Disparity Remains an Important Challenge for American Orthopedics
}

\author{
Charles N. Cornell, MD
}

Received: 10 August 2018/Accepted: 13 August 2018/Published online: 27 August 2018

(C) Hospital for Special Surgery 2018

Welcome to the final issue of the 14th volume of HSS Journal ${ }^{\circledR}$. This issue illustrates the value of our multidisciplinary mission, with articles covering a variety of topics and several that build on our efforts to improve care quality and patient outcomes after reconstructive orthopedic surgery.

Allow me to direct your attention to several notable contributions. Brusalis and colleagues provide an excellent review of a study highlighting the advantages of tranexamic acid administration to patients undergoing total knee replacement, and they address difficult questions surrounding the controversial use of tourniquets for this procedure. Marx and colleagues report on a crucial topic for reconstructive surgeons: the challenges of aligning the expectations of surgeon and patient regarding outcomes of anterior cruciate ligament reconstruction. It is clear that well-prepared patients generally achieve better outcomes and express greater satisfaction. Still, surgeons face challenges in educating their patients on the benefits and limitations of any surgical procedure. In this study, the authors report that expectations are well managed in most cases but point out scenarios that require the surgeon's special attention.

Perhaps the most compelling article presented is by Della Valle and his team. They review the early return of patients for inpatient or emergency care following total hip or total knee replacement. This topic has particular relevance in view of the current interest in moving arthroplasty into the ambulatory surgery arena. The researchers document the most common reasons why patients seek acute care in the 90 days after surgery and the patient groups at greatest risk. Notably, patients without private insurance, African Americans and Hispanics, those older than 85 years, and patients of lower socioeconomic status most frequently require acute care after lower-extremity arthroplasty.

These findings suggest to me that health care disparities persist in our specialty and argue for enhanced preparation of vulnerable patients undergoing elective arthroplasty in order to overcome what is likely a reversible disadvantage.

Please explore this excellent issue. As always, I welcome your feedback as well as your future manuscripts. It is my privilege to provide this forum for publication of your work.

\section{Compliance with Ethical Standards}

Conflict of Interest: Charles N. Cornell, MD, declares that he has no conflicts of interest.

Human/Animal Rights: N/A

Informed Consent: N/A

Required Author Forms Disclosure forms provided by the author are available with the online version of this article. 